\title{
Expanding treatment strategies for hepatocellular carcinoma patients: postoperative adjuvant transarterial chemoembolization
}

\author{
Rodolfo Sacco, Antonio Facciorusso \\ Gastroenterology Unit, Department of Medical Sciences, University of Foggia, Foggia, Italy \\ Correspondence to: Rodolfo Sacco, MD, PhD. Gastroenterology Unit, Department of Medical Sciences, University of Foggia, AOU Ospedali Riuniti, \\ Viale Pinto, 1, 71100 Foggia, Italy. Email: r.sacco@ao-pisa.toscana.it. \\ Comment on: Wang H, Du PC, Wu MC, et al. Postoperative adjuvant transarterial chemoembolization for multinodular hepatocellular carcinoma \\ within the Barcelona Clinic Liver Cancer early stage and microvascular invasion. Hepatobiliary Surg Nutr 2018;7:418-28.
}

Submitted Oct 02, 2019. Accepted for publication Nov 20, 2019.

doi: $10.21037 /$ hbsn.2019.11.33

View this article at: http://dx.doi.org/10.21037/hbsn.2019.11.33

In spite of the recent improvements in diagnostic and treatment options for hepatocellular carcinoma (HCC) patients, with an increasing number of patients diagnosed in early stage and offered more effective therapies, the high recurrence rates even after radical treatments, such as surgery or radiofrequency ablation, still represent an unsolved issue (1).

While neoadjuvant strategies are commonly adopted to induce downstaging of patients unsuitable to surgery or before orthotopic liver transplantation (OLT), adjuvant therapy aims at decreasing the recurrence rate after radical treatments.

In general, post-surgical recurrences of HCC are distinguished into early and late recurrences. Early recurrences (within 2 years from the curative treatment) are typically diagnosed within the same hepatic segment (or immediately adjacent to the location of the primary tumor) and are considered real recurrences (i.e., recurrences of the primary tumor), while late recurrences (beyond 2 years) are due to the chronic liver disease and the occurrence of $d e$ novo nodules (2).

In spite of the encouraging findings of some retrospective series and the potential advantages of sorafenib (Nexavar ${ }^{\circledR}$, Bayer, Leverkusen, Germany) as adjuvant agent, a large multicenter randomized-controlled trial (RCT) [Sorafenib as Adjuvant Treatment in the Prevention Of Recurrence of Hepatocellular Carcinoma (STORM)] recruiting 1,114 HCC patients after resection or RFA failed to show a significant improvement in survival outcomes (3). Likewise, other drugs determined conflicting results in the adjuvant setting because of their high cost and narrow therapeutic window $(4,5)$, while safer and inexpensive therapeutic agents seem promising but need to be tested in large RCTs $(6,7)$.

In an interesting manuscript recently published in HepatoBiliary Surgery and Nutrition, Wang et al. demonstrated that postoperative adjuvant transarterial chemoembolization (TACE) might represent a safe intervention able to effectively prevent tumor recurrence and improve overall survival in HCC patients classified in the Barcelona Clinic Liver Cancer (BCLC) early stage with multinodular disease and microvascular invasion (MVI) (8). The authors retrospectively reviewed their series of two hundred and seventy-one patients undergoing curative hepatectomy, of which $45.3 \%$ underwent adjuvant TACE and $47.2 \%$ were MVI positive (8). MVI and no use of adjuvant TACE resulted as independent risk factors for poor recurrence and survival outcomes in multivariate analysis (8).

In particular adjuvant TACE proved to be helpful in patients with MVI (5-year disease-free survival $26.3 \%$ vs. $20.7 \%, \mathrm{P}=0.038$ and 5 -year overall survival $73.6 \%$ vs. $47.7 \%, \mathrm{P}=0.005$ in patients undergoing TACE as compared to surgery alone, respectively), while no differences were noted in MVI negative patients with or without postoperative TACE (8).

TACE has been tested in the adjuvant setting after hepatic resection with discordant results. A phase III RCT enrolling 280 patients with HBV-related HCC found a significant recurrence and survival benefit in subjects undergoing TACE as compared to surgery alone (9). 
Nevertheless, a second RCT recruiting low-risk subjects did not confirm these findings (10).

Therefore, the first issue is to identify the accurate prognostic factors able to predict tumor recurrence after curative surgery. Undoubtedly, MVI represents an unequivocal predictor of recurrence but other molecular and clinical factors are needed in order to define the prognostic classes and to offer TACE only to patients who could really benefit from this treatment (11).

Although several retrospective studies, including the manuscript by Wang et al., show some benefits with adjuvant TACE treatment after resection in patients at high risk for recurrence (MVI or large tumors) $(8,12,13)$, RCTs are still rare and prospective evidence is needed to draw general assumptions in this field and to inform forthcoming clinical guidelines.

Furthermore, nearly the whole body of evidence supporting the use of postoperative adjuvant TACE is based on Eastern studies (who have usually different disease characteristics such as hepatitis B virus which is the main etiology in Asia but not in the Western countries), therefore the applicability of these findings in Western patients is still controversial.

Another open issue is the exact timing of TACE after surgery due to the risk of post-resection liver failure (14).

In conclusion, although the retrospective nature of the manuscript by Wang et al. cannot address adequately all of the aforementioned points, the authors should be commended for their efforts which help to put in perspective the use of adjuvant TACE in HCC patients after surgical resection.

\section{Acknowledgments}

None.

\section{Footnote}

Conflicts of Interest: The authors have no conflicts of interest to declare.

Ethical Statement: The authors are accountable for all aspects of the work in ensuring that questions related to the accuracy or integrity of any part of the work are appropriately investigated and resolved.

\section{References}

1. European Association for the Study of the Liver. EASL Clinical Practice Guidelines: Management of hepatocellular carcinoma. J Hepatol 2018;69:182-236.

2. Facciorusso A, Del Prete V, Antonino M, et al. Postrecurrence survival in hepatocellular carcinoma after percutaneous radiofrequency ablation. Dig Liver Dis 2014;46:1014-9.

3. Bruix J, Takayama T, Mazzaferro V, et al. Adjuvant sorafenib for hepatocellular carcinoma after resection or ablation (STORM): a phase 3, randomised, double-blind, placebo-controlled trial. Lancet Oncol 2015;16:1344-54.

4. Mazzaferro V, Romito R, Schiavo M, et al. Prevention of hepatocellular carcinoma recurrence with alpha-interferon after liver resection in $\mathrm{HCV}$ cirrhosis. Hepatology 2006;44:1543-54.

5. Hsu YC, Ho HJ, et al. Postoperative peg-interferon plus ribavirin is associated with reduced recurrence of hepatitis C virus-related hepatocellular carcinoma. Hepatology 2013;58:150-7.

6. Facciorusso A, Del Prete V, Crucinio N, et al. Angiotensin receptor blockers improve survival outcomes after radiofrequency ablation in hepatocarcinoma patients. J Gastroenterol Hepatol 2015;30:1643-50.

7. Pinter M, Weinmann A, Wörns MA, et al. Use of inhibitors of the renin-angiotensin system is associated with longer survival in patients with hepatocellular carcinoma. United European Gastroenterol J 2017;5:987-96.

8. Wang H, Du PC, Wu MC, et al. Postoperative adjuvant transarterial chemoembolization for multinodular hepatocellular carcinoma within the Barcelona Clinic Liver Cancer early stage and microvascular invasion. Hepatobiliary Surg Nutr 2018;7:418-28.

9. Wang Z, Ren Z, Chen Y, et al. Adjuvant Transarterial Chemoembolization for HBV-Related Hepatocellular Carcinoma After Resection: A Randomized Controlled Study. Clin Cancer Res 2018;24:2074-81.

10. Zhai XF, Liu XL, Shen F, et al. Traditional herbal medicine prevents postoperative recurrence of small hepatocellular carcinoma: A randomized controlled study. Cancer 2018;124:2161-8.

11. Akateh C, Black SM, Conteh L, et al. Neoadjuvant and adjuvant treatment strategies for hepatocellular carcinoma. 
World J Gastroenterol 2019;25:3704-21.

12. Peng BG, He Q, Li JP, et al. Adjuvant transcatheter arterial chemoembolization improves efficacy of hepatectomy for patients with hepatocellular carcinoma and portal vein tumor thrombus. Am J Surg 2009;198:313-8.

13. Ye JZ, Chen JZ, Li ZH, et al. Efficacy of postoperative adjuvant transcatheter arterial chemoembolization in

Cite this article as: Sacco R, Facciorusso A. Expanding treatment strategies for hepatocellular carcinoma patients: postoperative adjuvant transarterial chemoembolization. Hepatobiliary Surg Nutr 2020;9(1):59-61. doi: 10.21037/ hbsn.2019.11.33 hepatocellular carcinoma patients with microvascular invasion. World J Gastroenterol 2017;23:7415-24.

14. Citterio D, Facciorusso A, Sposito C, et al. Hierarchic Interaction of Factors Associated With Liver Decompensation After Resection for Hepatocellular Carcinoma. JAMA Surg 2016;151:846-53. 